Programming Techniques

M. Douglas McIlroy*

and Data Structures

Editor

Distributed Computation on Graphs:

\section{Shortest Path Algorithms}

\author{
K. M. Chandy and J. Misra \\ University of Texas at Austin
}

We use the paradigm of diffusing computation, introduced by Dijkstra and Scholten, to solve a class of graph problems. We present a detailed solution to the problem of computing shortest paths from a single vertex to all other vertices, in the presence of negative cycles.

CR Categories and Subject Descriptors: C.2.4 [Computer-Communication Networks]: Distributed Systems; D.1.3 [Programming Techniques]: Concurrent Programming; F.2.2 [Analysis of Algorithms and Problem Complexity]: Nonnumerical Algorithms and Problems; G.2.2 [Discrete Mathematics]: Graph Theory

General Terms: Algorithm, Theory

Additional Key Words and Phrases: distributed computation, shortest path, negative cycle, depth first search, diffusing computation

\section{Introduction}

This paper presents distributed algorithms based on the work of Dijkstra and Scholten [1], for solving graph problems using networks of communicating processes. The solution to one particular graph problem, that of finding shortest paths from a single vertex to all other vertices in a weighted, directed graph, in the presence of negative cycles, is discussed in detail. We then show how this solution may be applied to other graph problems including depth-first search in an undirected graph.

* Former editor of Programming Techniques and Data Structures, of which Ellis Horowitz is the current editor.

This work was supported in part by the Air Force Office of Scientific Research under grant AFOSR 81-0205.

Authors' Present Address: K. Mani Chandy and J. Misra, Computer Sciences Department, University of Texas, Austin, TX 78712.

Permission to copy without fee all or part of this material is granted provided that the copies are not made or distributed for direct commercial advantage, the ACM copyright notice and the title of the publication and its date appear, and notice is given that copying is by permission of the Association for Computing Machinery. To copy otherwise, or to republish, requires a fee and/or specific permission. (c) 1982 ACM 0001-0782/82/1100-0833 $\$ 00.75$.
Our model of computation is a network of processes in which processes communicate only by sending and receiving messages; the model is presented in detail in Sec. 2. We describe the classical shortest path problem [2] and the necessary terminology from graph theory in Sec. 3. The distributed algorithm is given in Sec. 4 and its proof in Sec. 5. Applications to other graph problems are discussed in Sec. 6.

\section{Model of a Network of Communicating Processes}

A process is a sequential program which can communicate with other processes by sending/receiving messages. Two processes $P$ and $Q$ are said to be neighbors if they can communicate directly with one another without having messages go through intermediate processes. We assume that communication channels are bidirectional: if $P$ can send messages to $Q$ then $Q$ can send messages to $P$. A process knows the identities of its neighbors; otherwise it is ignorant of the identities of all other processes and of the general structure of the network.

We assume a very simple protocol for message communication; this protocol is equivalent to the one used by Dijkstra and Scholten [1]. Every process has an input buffer of unbounded length. If process $P$ sends a message to a neighbor process $Q$, then the message gets appended at the end of the input buffer of $Q$ after a finite, arbitrary delay. We assume that (1) messages are not lost or altered during transmission, (2) messages sent from $P$ to $Q$ arrive at $Q$ 's input buffer in the order sent, and (3) two messages arriving simultaneously at an input buffer are ordered arbitrarily and appended to the buffer. A process receives a message by removing one from its input buffer.

The assumption of unbounded length buffers is for ease of exposition. We show, in Sec. 6, that for our problem the input buffer length of process $Q$ can be bounded by the number of neighbors of $Q$.

\section{The Shortest Path Problem}

$G=(V, E)$ is a directed graph in which $V$ is the set of vertices and $E$ is the set of edges. Edge $\left(v_{i}, v_{j}\right)$ has an associated length $w_{i j}$. If edge $\left(v_{i}, v_{j}\right)$ exists then $v_{j}$ is said to be a successor of $v_{i}$ and $v_{i}$ is said to be a predecessor of $v_{j}$. It is required to determine lengths of the shortest paths from a special vertex $v_{1}$ in $V$ to all other vertices in $V^{1}$ Since some $w_{i j}$ may be negative, a cycle of negative total length (called a negative cycle) may exist in the graph. If a negative cycle is reachable from $v_{1}$ then all vertices reachable from that negative cycle will have a shortest path length of $-\infty$. The distance of a vertex $v_{i}$ is the length of the shortest path from $v_{1}$ to $v_{i}$ and is denoted by $L_{i}$.

\footnotetext{
${ }^{1}$ We assume familiarity with graph theoretic terms such as path, shortest path, etc.

Communications

of

the ACM

November 1982

Volume 25

Number 11
} 


\section{A Distributed Algorithm for the Shortest Path Problem}

Consider a network of processes corresponding to graph $G$; process $p_{i}$ represents vertex $v_{i}$, for all $i$, and $p_{i}$ and $p_{j}$ are neighbors if edges $\left(v_{i}, v_{j}\right)$ or $\left(v_{j}, v_{i}\right)$ exist in $G$. $p_{i}$ knows the weight $w_{i j}$ for every outgoing edge ( $v_{i}$, $\left.v_{j}\right)$. However, $p_{i}$ may not know the weights of incoming edges or the identities of processes other than its neighbors.

Process $p_{1}$ initiates a computation to determine the lengths of shortest paths from $v_{1}$ to all vertices. In the following, we use vertex $v_{i}$ and process $p_{i}$ interchangeably when no confusion can result.

\subsection{The Structure of the Algorithm}

The algorithm works in two phases, both of which are initiated by $p_{1}$. At the end of phase $I$, every process $p_{i}$ will have the value of $L_{i}$, if $L_{i} \neq-\infty$. If for some vertex $v_{j}, j \neq 1, L_{j}=-\infty$ then $p_{j}$ will not be aware of this fact at the end of phase I; the goal of phase II is to inform all such processes that they are at distances of $-\infty$.

\subsection{The Structure of Phase I Computation}

\subsubsection{Messages Used in Phase I}

Phase I computation uses two kinds of messages:

(1) A length message is a two-tuple $(s, p)$, where $p$ is the identity of the process sending the message and $s$ is a number. $p_{i}$ sends a length message $\left(s, p_{i}\right)$ to $p_{j}$ to inform $p_{j}$ that there is a path of length $s$ from $v_{1}$ to $v_{j}$ in which $v_{i}$ is the prefinal vertex.

(2) An acknowledgment message or ack has no other data associated with it. A process $p_{j}$ sends an ack to a process $p_{i}$ in response to a length message sent by $p_{i}$. Intuitively, an ack denotes that the length sent by $p_{i}$ to $p_{j}$ has been (or will be) taken into consideration by all processes reachable from $p_{j}$.

A process $p_{i}, i \neq 1$, maintains a local variable $d$ which denotes the length of the shortest path received so far by $p_{i}$. Upon receiving a length $s$ from a predecessor, if $s<$ $d, p_{i}$ sets $d$ to $s$ and in this case it sends a length message $\left(s+w_{i j}, p_{i}\right)$ to every successor $p_{j}$. It may seem that acks are superfluous. Clearly length messages can be used to compute successively shorter paths. However, the presence of negative cycles means that this will be a nonterminating computation. Acks are used to terminate phase I computation as described below.

\subsubsection{Local Data Used by a Process $p_{i}$ During Phase I}

Each process $p_{i}$ uses three local variables:

d This is the shortest length of paths from $v_{1}$ to $v_{i}$ known to this process at this point in the computation; $d=\infty$ if no length message has been received.

pred This is the predecessor from which the length $d$ was received; this is the prefinal vertex on the shortest path to $v_{i}$ computed so far. pred is undefined if $d=\infty$ or $i=1$.

num This is the number of unacknowledged messages, that is, the number of messages sent by this process for which no ack has been received so far.

\subsubsection{Phase I Algorithm for Process $p_{j}, j \neq 1$ \\ Initialization}

(no length message has been received; there are no unacknowledged messages

begin $d:=\infty ;$ pred is undefined; num :=0 end;

Upon receiving a length message $\left(s, p_{i}\right)$

if $s<d$ then

begin

(send an ack to pred, the prefinal vertex on the previous shortest path, if it has not been sent already

if num $>0$ then send an ack to pred;

\{update $d$, pred \}

pred $:=p_{i} ; d:=s$

(send length messages to all successors of $v_{j}$ and increment num appropriately and then return ack to pred if num $=0$ \}

send a length message $\left(d+w_{j k}, p_{j}\right)$ to every successor $p_{k}$; num $:=$ num + the number of successors of $v_{j}$;

if num $=0$ then send an ack to pred

end

else $\{s \geq d$ \} (new length does not denote a shorter path) send ack to $p_{i}$.

Upon receiving an ack from process $p_{h}$

begin

[decrement number of unacknowledged messages\}

num := num - 1;

\{send acknowledgement to pred if acks have been received for all messages

if $n u m=0$ then send ack to pred

end.

Note. If num $>0$ at any time, then a process has exactly one message to which it has not sent an ack, and this ack should go to pred.

\subsubsection{Initiation of Phase I}

\subsubsection{Phase I algorithm for process $p_{1}$}

\section{Initialization}

$d:=0 ;$ pred is undefined;

send $\left(w_{1 k}, p_{1}\right)$ to all successors $p_{k} ;$ num $:=$ number of successors of $v_{1}$.

Upon receipt of a length message $\left(s, p_{i}\right)$

(start phase II if a negative cycle is detected \}

if $s<0$ then terminate phase I and start phase II else return ack to $p_{i}$

Upon receiving an ack

(update num; start phase II if there is no unacknowledged message remaining

num := num -1 ;

if num $=0$ then terminate phase I and start phase II.

\subsubsection{Example}

Consider the graph shown in Figure 1. Four feasible snapshots of the network showing possible values for $d$, pred, and num for the six processes in this example are shown below. Since transmission delays are arbitrary, network computation is nondeterministic. Hence the four

$\begin{array}{ll}\begin{array}{l}\text { Communications } \\ \text { of }\end{array} & \begin{array}{l}\text { November } 1982 \\ \text { Volume 25 } \\ \text { the ACM }\end{array} \\ \text { Number 11 }\end{array}$


snapshots shown below form only one of many sequences which may arise. The question mark denotes an undefined value for $p$ red.

Snapshot 1. $p_{1}$ has sent one message to each of $p_{2}$ and $p_{3}$ which have not yet been received.

\begin{tabular}{c|c|c|c|c|c|c|}
\multirow{4}{*}{$\begin{array}{c}d \\
\text { pred }\end{array}$} & 1 & 2 & 3 & 4 & 5 & 6 \\
\cline { 2 - 7 } num & 0 & $\infty$ & $\infty$ & $\infty$ & $\infty$ & $\infty$ \\
\cline { 2 - 7 } & $?$ & $?$ & $?$ & $?$ & $?$ & $?$ \\
\hline & 2 & 0 & 0 & 0 & 0 & 0 \\
\hline
\end{tabular}

Snapshot 2. $p_{2}, p_{3}$ have received length messages $(3$, $\left.p_{1}\right),\left(4, p_{1}\right)$, respectively. $p_{3}$ has sent $\left(10, p_{3}\right)$ to $p_{4}$, which $p_{4}$ has received.

\begin{tabular}{|c|c|c|c|c|c|c|}
\multirow{4}{*}{$\begin{array}{c}d \\
\text { pred }\end{array}$} & \multicolumn{1}{c}{1} & \multicolumn{1}{c}{2} & 3 & 4 & 5 & 6 \\
\cline { 2 - 7 } num & 0 & 3 & 4 & 10 & $\infty$ & $\infty$ \\
\cline { 2 - 7 } & 2 & 1 & 1 & 3 & $?$ & $?$ \\
\hline & 2 & 0 & 1 & 0 & 0 & 0 \\
\hline
\end{tabular}

Snapshot 3. $p_{5}, p_{6}$ receive $\left(11, p_{4}\right),\left(12, p_{4}\right)$, respectively, from $p_{4} . p_{6}$ sends an ack to $p_{4}$; this ack is received by $p_{4}$. $p_{4}$ receives $\left(5, p_{2}\right)$. Next $p_{4}$ sends an ack to $p_{3}$, which is received, and sends $\left(6, p_{4}\right),\left(7, p_{4}\right)$ to $p_{5}$ and $p_{6}$, respectively, which they both receive. $p_{5}$ sends an ack to $p_{4}$ which is received by $p_{4}$.

\begin{tabular}{|c|c|c|c|c|c|c|}
\hline & 1 & 2 & 3 & 4 & 5 & 6 \\
\hline$d$ & 0 & 3 & 4 & 5 & 6 & 7 \\
\hline pred & $?$ & 1 & 1 & 2 & 4 & 4 \\
\hline num & 2 & I & 0 & 2 & 0 & 0 \\
\hline
\end{tabular}

Snapshot 4. $p_{3}$ sends an ack to $p_{1}$ since $p_{3}$ 's num is zero. $p_{5}$ sends $\left(2, p_{5}\right)$ to $p_{2}$, thus causing $p_{2}$ to send an ack to $p_{1}$. The acks are received since $p_{1}$ has no further unacknowledged messages it terminates phase $I$.

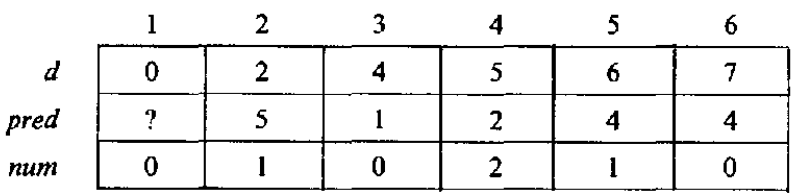

\subsection{The Structure of Phase II Computation}

\subsubsection{Messages Used in Phase II}

Phase II employs two kinds of messages: over? and over-. An over- message is sent by process $j$ to all its successors if process $j$ has determined that phase $\mathrm{I}$ is over and $L_{j}=-\infty$; an over- message orders the recipient to halt all phase I computation (if it has not done so already), set its $d$ to $-\infty$ and propagate the over- message to its successors. If a process already has its $d=-\infty$ when it receives an over- message, it takes no action. An over? message is sent by process $j$ to all its successors when it has determined that phase $I$ is over, but has not determined whether $L_{j}=-\infty$. An over? message orders the recipient to halt all phase I computation. If the recipient $p_{i}$ has num $=0$ it sends over? messages to its successors; otherwise (if $p_{i}$ has num $>0$ ) it can be shown
Fig. 1. A Network with Weighted Edges.

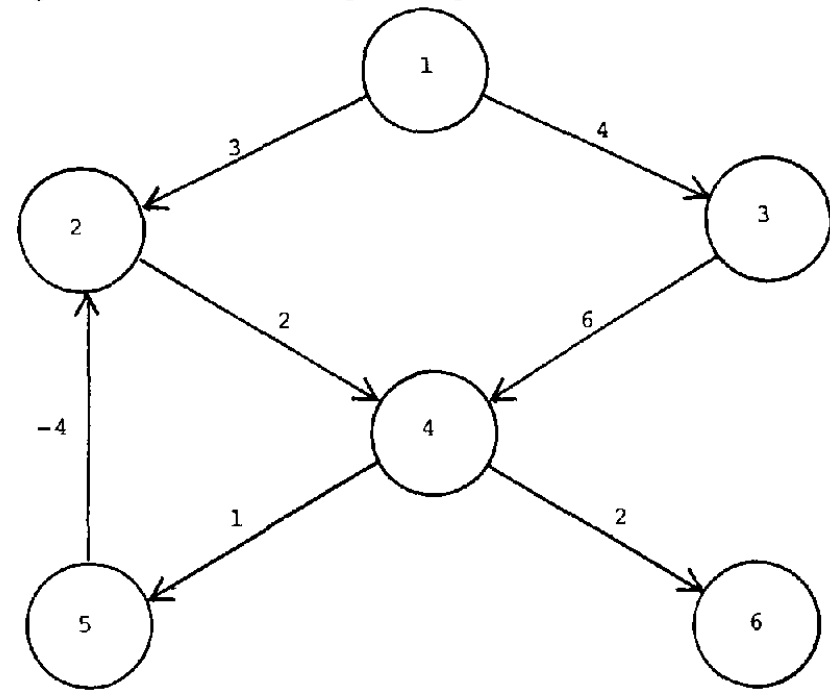

that $L_{i}=-\infty$, and therefore $p_{i}$ sets its $d:=-\infty$ and sends over- to its successors. Note that it is redundant for any process $p_{i}$ to send duplicate messages to a process $p_{j}$ or to send over? after over-. Every process other than $p_{1}$ will receive an over? or an over- message.

\subsubsection{Detailed Algorithm for Phase II}

\subsubsection{Initiation of Phase II by Process $p_{1}$}

if $p_{1}$ receives a message $(s, p)$, with $s<0$, during phase $\mathrm{I}$

then $\left\{p_{1}\right.$ detects that it is in a negative cycle

send an over-message to all its successors

else $\left\{\right.$ num $=0$ for $p_{1}$ at the end of phase 1 \}

send over? message to all successors.

\subsubsection{Phase II Algorithm for Process $p_{j}, j \neq 1$ with} num $_{j}>0$

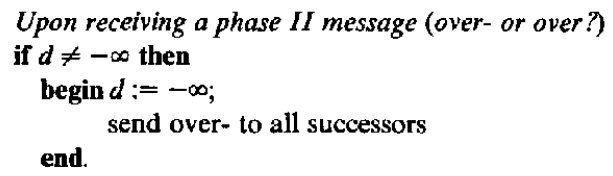

\subsubsection{Phase II Algorithm for Process $p_{j}, j \neq 1$ with} num $_{j}=0$

Upon receiving an over- message

if $d \neq-\infty$ then

begin $d:=-\infty$; send over- to all successors

end.

Upon receiving an over? message

if $d \neq-\infty$ then send over? to all successors who have not been sent such a message.

\section{Proof of Correctness}

We define $v_{i}$ to be a finite vertex if $L_{i} \neq-\infty ; v_{i}$ is an infinite vertex if $L_{i}=-\infty$.

LEMMA 1. For any $j, L_{j} \leq d_{j}$ at all times.

Proof. We observe that every $d_{j}$ is the length of some path from $v_{1}$ to $v_{j}$.
Communications

of

the ACM
November 1982

Volume 25

Number 11 
LEMMA 2. If there is a finite path of length $d_{j}^{*}$ to a vertex $v_{j}$, then from some point onward in the computation $d_{j} \leq d_{j}^{*}$, if phase I does not terminate.

Proof. Proof is by induction on the number of edges on the path. Lemma 2 is trivial when the number of edges in the path is zero. Now assume Lemma 2 holds for all paths with $k$ or fewer edges. Consider a path with $k+1$ edges from $v_{1}$ to $v_{j}$ in which $v_{i}$ is the prefinal vertex and the path length to $v_{i}$ is $d_{i}^{*}=d_{j}^{*}-w_{i j}$. From the induction hypothesis eventually, $d_{i} \leq d_{i}^{*}=d_{j}^{*}-w_{i j}$; therefore $p_{j}$ will eventually receive $\left(d_{i}+w_{i j}, p_{i}\right)$ which guarantees that $d_{j} \leq d_{i}+w_{i j} \leq d_{j}^{*}$. It follows from the algorithm that $d_{j}$ can never increase. Therefore, $d_{j} \leq d_{j}^{*}$ from that point onward in the computation.

LEMMA 3. If phase I does not terminate then from some point onward in the computation, every infinite vertex $v_{j}$ will have an infinite vertex for pred $d_{j}$ and every finite vertex $v_{j}$ will have a finite vertex for pred ${ }_{j}, j \neq 1$. times:

Proof. The following holds for all $j, j \neq 1$, at all

$$
d_{i}+w_{i j} \leq d_{j} \quad \text { if } i=\text { pred }_{j} .
$$

From Lemma $1, L_{i} \leq d_{i}$, for all $i$. Therefore,

$$
L_{i}+w_{i j} \leq d_{j}, \quad \text { if } i=\text { pred }_{j} .
$$

If $v_{j}$ is infinite then from Lemma 2 , eventually $d_{j}$ gets arbitrarily small. In particular, from some point onward in the computation, for every finite $v_{i}$,

$$
d_{j}<L_{i}+w_{i j} .
$$

Hence from that point onward pred $_{j}$ will be an infinite vertex.

From Lemmas 1 and 2, if phase I does not terminate then eventually every finite $v_{i}$ will have $d_{i}=L_{i}$ and pred $d_{i}$ will be the prefinal vertex on this path; pred $_{i}$ must therefore be a finite vertex.

\section{THEOREM 1. Phase I terminates.}

Proof. Assume phase I never terminates. Then $d_{j}$ $=L_{j}$ for every finite vertex $v_{j}$ from some point in phase I computation and hence no finite vertex sends a length message from then on. From Lemma 3, finite vertices eventually form a rooted directed tree where pred $;$ is the father of $v_{j}, j \neq 1$, and $v_{1}$ is the root. A leaf vertex $v_{j}$, $j \neq 1$, in this tree cannot be the pred for any finite vertex (since it is a tree) nor can it be the pred for any infinite vertex, from Lemma 3; therefore eventually $n \boldsymbol{m}_{j}=0$ and $v_{j}$ will send an ack to pred $d_{j}$. Induct on the height of the tree to show that every finite vertex will eventually have num $=0$. If $p_{1}$ is a finite vertex it will then terminate phase I computation. If $p_{1}$ is an infinite vertex, from Lemma 2, it will eventually detect that it is in a negative cycle and hence terminate phase I. Hence phase I will terminate! Contradiction!

836
Theorem 2. At the termination of phase I,

(1) if $v_{j}$ is a finite vertex, $d_{j}=L_{j}$ and num $\dot{m}_{j}=0$;

(2) if $v_{j}$ is an infinite vertex, then and only then, there is some $v_{i}$ such that there is a path from $v_{1}$ to $v_{j}$ through $v_{i}$, in the graph, and num $i>0$.

Proof. (1) For a finite vertex $v_{j}$, we define $e(j)$ to be the number of edges on a shortest path from $v_{1}$ to $v_{j}$ (if there are several shortest paths we choose the shortest loop-free path with maximum number of edges). The result follows by induction on all vertices $v_{j}$ with $e(j) \leq$ $k$, for $k=0,1,2 \ldots$

(2) Assume the contrary that for an infinite vertex $v_{j}$, every vertex $v_{i}$ on a path from $v_{1}$ to $v_{j}$ has $n u m_{i}=0$, at the end of phase I. Even if $p_{1}$ did not terminate phase I computation, $v_{j}$ will never receive a length message and thus $d_{j}$ will not decrease. This contradicts Lemma 2 . The other part of the proof follows by similar arguments.

THEOREM 3. Phase II terminates and at that point $d_{j}=L_{j}$ for every vertex $v_{j}$.

Proof. Phase II terminates since any process sends at most 2 messages: over? followed by an over- message. No finite vertex receives an over-message because there cannot be an infinite vertex on a path from $v_{1}$ to a finite vertex. Therefore $d_{j}$ remains unchanged during phase II for a finite vertex; and from Theorem $2, d_{j}=L_{j}$ at the beginning of phase II. For an infinite vertex $v_{j}$, there is a path from $v_{1}$ to $v_{j}$ through $v_{i}$, where num $i>0$ at the end of phase I. Therefore $p_{i}$ will propagate an overmessage once it receives any phase II message, and therefore $d_{j}=-\infty=L_{j}$ eventually.

\section{Notes on the Algorithm}

\subsection{Unbounded Buffers}

A process $p_{i}$ sends (strictly) monotone decreasing lengths in every length message to any other process $p_{j}$. Therefore any length message sent by $p_{i}$ can overwrite any earlier message sent by $p_{i}$ which is still in the buffer. Hence $p_{j}$ need only store one message (the latest message) from each predecessor. The space requirement for acks can be reduced by storing the number of acks sent from $p_{j}$ to $p_{i}$, which are still in the buffer; this number is incremented by 1 each time $p_{j}$ sends an ack to $p_{i} . p_{i}$ can remove multiple acks from the buffer and reduce num $_{i}$ accordingly. Hence we need space for at most one message and one ack count for every neighbor of a process $p_{j}$ in the input buffer of $p_{j}$.

\subsection{Applications to Other Graph Problems}

A number of other graph problems can be formulated as shortest path problems using a more general notion of path length. We define a path length function $f$, a real valued function on paths, starting from $v_{1}$, as follows:

$\begin{array}{ll}\text { Communications } & \text { November } 1982 \\ \text { of } & \text { Volume 25 } \\ \text { the ACM } & \text { Number } 11\end{array}$




$$
\begin{aligned}
& f[\text { path with no edges }]=0 \\
& f\left[P_{i} ;(i, j)\right]=g_{i}\left(f\left(P_{i}\right), w_{i j}\right),
\end{aligned}
$$

where $P_{i}$ is any path from $v_{1}$ to $v_{i}, P_{i} ;(i, j)$ is the path $P_{i}$ followed by edge $\left(v_{i}, v_{j}\right), g_{i}$ is any arbitrary computable function which is monotone in the first argument, and $w_{i j}$ is some given real number denoting the "length" of edge $\left(v_{1}, v_{j}\right)$.

The shortest path algorithm of Sec. 4 can be used to compute

$d_{j}=\min \left\{f\left(P_{j}\right) \mid P_{j}\right.$ is a path from $v_{1}$ to $\left.v_{j}\right\}, \quad$ for all $j$.

The only change is in phase I computation in the content of the length message sent; instead of $p_{j}$ sending $\left(d_{j}+w_{j k}, p_{j}\right)$ to a successor $p_{k}$, it now sends $\left(g_{j}\left(d_{j}, w_{j k}\right)\right.$, $\left.p_{j}\right)$ ). Monotonicity of $g$ in the first argument is essential, since it guarantees that every process sends monotone decreasing path lengths, if it receives monotone decreasing path lengths.

We list some graph problems and show how they can be solved under this shortest path formulation.

(1) Find all vertices reachable from $v_{i}$. We wish to set $d_{j}$ to 0 if $v_{j}$ is reachable from $v_{1}$; else set $d_{j}$ to $\infty$. We use the following function,

$$
g_{i}(x, y)=x \text {. }
$$

(2) Find all vertices which can reach $v_{1}$. This is the same as (1), except length messages are sent to predecessors.

(3) Find the maximum strongly connected component. Determine if a given vertex $v_{1}$ is in a nontrivial strongly connected component: use both (1) and (2). A separate computation is then needed to determine whether there is a vertex which has its $d$ set to 0 in both computations.

(4) Construct a depth-first search tree. Consider an undirected graph $G$. For each vertex $j$ label all the edges incident on $j$ with $1,2,3, \ldots$ In a depth-first search we would normally label the "left-most" edge on $j$ with 1 , the next left-most edge 2, and so on. (However, for purposes of proof the labeling is arbitrary.) Note that edge $(i, j)$ may be the $r$ th left-most edge incident on $i$ and the sth left-most edge incident on $j$ and it is not necessary that $r=s$. An example is shown below.

In a depth-first search starting from a vertex (say vertex 1), the vertices of the graph are traversed beginning with a depth-first search of the left-most successor of vertex 1. The collection of paths traversed to reach each vertex for the first time forms a tree called the depth-first search tree. In the above example the depthfirst search tree has edges $(1,2),(2,3)$, and $(3,4)$. Our goal is to determine the depth-first search tree; in particular we want to determine the path leading to every vertex in the depth-first search tree.
Fig. 2. An Undirected Graph with Labeled Edges: An Application of Depth-First Search.

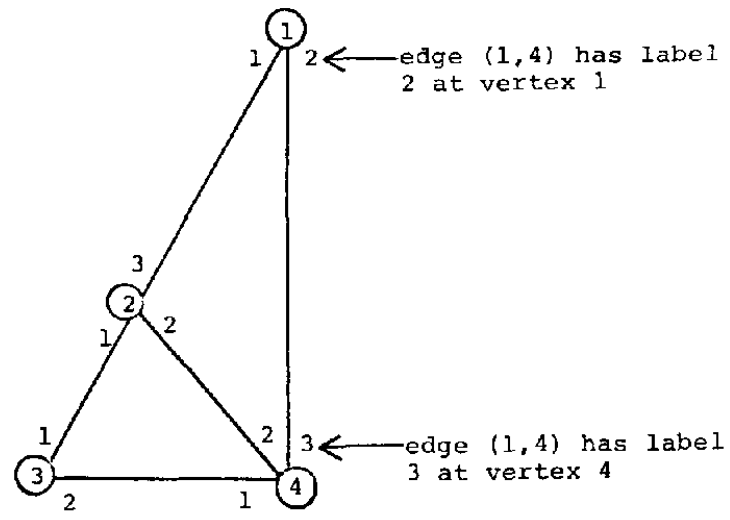

Let $P$ be a path $\left(i_{1}, \ldots, i_{k}\right)$. Then define $\varphi(P)=$ $\left(j_{1} \ldots, j_{k-1}\right)$, where $j_{m}, m=1, \ldots, k-1$, is the label assigned to edge $\left(i_{m}, i_{m+1}\right)$ at vertex $i_{m}$. In our example, if $P=(1,2,3,4)$ then $f(P)=(1,1,2)$.

Let $f(P)=\left(j_{1}, \ldots, j_{m}\right)$ and $f\left(P^{\prime}\right)=\left(k_{1}, \ldots, k_{n}\right)$. We define $f(P)<f\left(P^{\prime}\right)$ if and only if either

(i) for some $r, j_{r}<k_{r}$ and $j_{i}=k_{i}$ for $i=1, \ldots, r-1$, or

(ii) $n>m$ and $j_{i}=k_{i}$ for $i=1, \ldots, m$.

Thus $(1,2,3)<(3)$ and $(1)<(1,1,2,2)$.

It is evident that $d_{j}=\min \left\{f\left(P_{j}\right) \mid P_{j}\right.$ is a path from $v_{1}$ to $v_{j}$ \} denotes the path in the depth-first search tree up to $v_{j}$.

\subsection{Earlier Work}

The algorithm suggested in this paper is a modification of an algorithm proposed by Dijkstra and Scholten [1] for termination detection of a class of distributed computations, called diffusing computations. In their algorithm pred does not change as long as num $_{j}>0$; the algorithm terminates when $n u m_{j}=0$ for every $p_{j}$. We allow pred $d_{j}$ to change while num $_{j}>0$; this allows us to terminate the phase I algorithm even when some num $>0$. This is critical for identifying infinite vertices since those are the ones which are reachable from a vertex with num $>0$.

Acknowledgments. We are indebted to E. W. Dijkstra for his comments on an earlier draft of this paper; his suggestions led to more concise proofs in Section 5 . We are also grateful to unknown referees and M. D. McIlroy for their suggestions and corrections.

Received 7/80; revised 9/81; accepted 3/82

References

1. Dijkstra, E.W., and Scholten, C.S. Termination detection for diffusing computations. Inf. Process. Lett 11, (Aug. 1980), 1, 1-4. 2. Ford, L.R., and Fulkerson, D.R. Flows in Networks. Princeton Univ. Press, Princeton, N. J., 1962.
Communications of the $\mathrm{ACM}$
November 1982

Volume 25

Number 11 